\title{
Evaluation of the heart and lung dosimetric parameters in deep inspiration breath hold using 3D Slicer
}

\author{
Azam Eskandari ${ }^{1}$, Shahrokh Nasseri ${ }^{1,2}$, Hamid Gholamhosseinian ${ }^{1,2}$, Sare Hosseini ${ }^{3}$, \\ Mohammad Javad Keikhai Farzaneh ${ }^{4}$, Alireza Keramati ${ }^{5}$, Maryam Naji ${ }^{6}$, Atefeh Rostami ${ }^{7}$, Mehdi Momennezhad ${ }^{1,8}$ \\ ${ }^{1}$ Department of Medical Physics, Faculty of Medicine, Mashhad University of Medical Sciences, Mashhad, Iran \\ ${ }^{2}$ Medical Physics Research Center, Mashhad University of Medical Sciences, Mashhad, Iran \\ ${ }^{3}$ Cancer Research Center, Mashhad University of Medical Sciences, Mashhad, Iran \\ ${ }^{4}$ Department of Medical Physics, Faculty of Medicine, Zahedan University of Medical Sciences, Zahedan, Iran \\ ${ }^{5}$ Student Research Committee, Mashhad University of Medical Sciences, Mashhad, Iran \\ ${ }^{6}$ Department of Radiation Oncology, Imam Reza Hospital, Mashhad University of Medical Sciences, Mashhad, Iran \\ ${ }^{7}$ Department of Medical Physics and Radiological Sciences, Sabzevar University of Medical Sciences, Sabzevar, Iran \\ ${ }^{8}$ Nuclear Medicine Research Center, Mashhad University of Medical Sciences, Mashhad, Iran
}

Received: December 27, 2019

Revised: February 29, 2020

Accepted: March 6, 2020

\section{Correspondence:}

Mehdi Momennezhad, PhD

Nuclear Medicine Research Center,

Mashhad University of Medical

Sciences, Pardis-e-daneshgah, Vakil

Abad Blvd, Mashhad, Iran

Tel: +985138002328

Fax: +98 5138002328

Email: momennezhadm@mums.ac.ir

ORCID:

http://orcid.org/0000-0002-1509-0446
Purpose: The present study was conducted to compare dosimetric parameters for the heart and left lung between free breathing (FB) and deep inspiration breath hold (DIBH) and determine the most important potential factors associated with increasing the lung dose for left-sided breast radiotherapy using image analysis with 3D Slicer software.

Materials and Methods: Computed tomography-simulation scans in FB and DIBH were obtained from 17 patients with left-sided breast cancer. After contouring, three-dimensional conformal plans were generated for them. The prescribed dose was 50 Gy to the clinical target volume. In addition to the dosimetric parameters, the irradiated volumes and both displacement magnitudes and vectors for the heart and left lung were assessed using 3D Slicer software.

Results: The average of the heart mean dose $\left(D_{\text {mean }}\right)$ decreased from 5.97 to 3.83 Gy and $V_{25}$ from $7.60 \%$ to $3.29 \%$ using DIBH ( $p<0.001$ ). Furthermore, the average of $D_{\text {mean }}$ for the left lung was changed from 8.67 to $8.95 \mathrm{~Gy}(p=0.389)$ and $V_{20}$ from $14.84 \%$ to $15.44 \%(p=0.387)$. Both of the absolute and relative irradiated heart volumes decreased from 42.12 to $15.82 \mathrm{~mL}$ and $8.16 \%$ to $3.17 \%$, respectively $(p<0.001)$; however, these parameters for the left lung increased from 124.32 to $223.27 \mathrm{~mL}(\mathrm{p}<0.001)$ and $13.33 \%$ to $13.99 \%(\mathrm{p}=0.350)$. In addition, the average of heart and left lung displacement magnitudes were calculated at 7.32 and $20.91 \mathrm{~mm}$, respectively. Conclusion: The DIBH is an effective technique in the reduction of the heart dose for tangentially treated left sided-breast cancer patients, without a detrimental effect on the left lung.

Keywords: Breath holding, Unilateral breast neoplasms, Heart, Radiotherapy, Conformal

\section{Introduction}

Radiotherapy after breast-conserving surgery removes microscopic disease in the conserved breast; therefore, it diminishes the risk of local recurrence and distant metastasis among patients. As a result, it improves the survival rate among them [1]. However, despite its benefit in the reduction of cancer-specific mortality, radiotherapy is known to cause cardiovascular disease and lung cancer, especially in patients with left-sided breast cancer $[2,3]$. The rate of ischemic heart disease increases linearly by $7.4 \%$ for each increase of 1 Gy in the mean heart dose [4]. As there is no threshold dose below which the side effects cannot occur; therefore, in recent years, 
there has been a lot of efforts to decrease the delivered dose to the organ at risks (OARs) as much as possible [2,4-6].

Deep inspiration breath hold (DIBH) is one of the methods used for the above-mentioned purpose. It is a simple and effective technique in decreasing the heart dose without compromising the coverage of target volume in patients with left-sided breast cancer [5]. In DIBH, the heart simply moves posteriorly and inferiorly due to lung expansion and diaphragmatic movement; as a result, the distance between the heart and irradiated areas, such as the breast and chest, increased leading to a reduction in the irradiated heart volume and its dose $[2,7,8]$. Although this technique reduces the heart dose [7-12], it seems that there are challenges in the reduction of lung dose with $\mathrm{DIBH}$ [13]. Despite the results of many studies showing the reduction of the ipsilateral lung dose $[2,7,11,14]$, there are reports of no significant difference and even increasing the left lung dosimetric parameters using DIBH [8,15-20].

Therefore, the primary aim of this study was to compare the dosimetric parameters of the heart and left lung between free breathing (FB) and DIBH. In the second place, the present study determined the most important potential factors associated with increasing the lung dose in left-sided breast radiotherapy with the calculation of various parameters, such as the irradiated volume and displacement of the lung, during DIBH in patients with left-sided breast cancer using image analysis with 3D Slicer software (version 4.10.1, Brigham and Women's Hospital, Boston, MA, USA) [21].

\section{Materials and Methods}

\section{Patient selection}

This study was carried out on 17 patients with early left-sided breast cancer who referred for postoperative radiotherapy following undergoing breast-conserving surgery during 2017-2019. The subjects enrolled in the present study voluntarily and signed the informed consent before the computed tomography (CT) simulation. These patients should be capable of holding their breath for at least 20 seconds.

This study was approved by the Institutional Review Board of Mashhad University of Medical Sciences (No. 970830).

\section{CT scan}

In the CT simulation, two scans were acquired with $5 \mathrm{~mm}$ slice thickness that was the first scan in FB and another in the DIBH state. The patients were scanned in the supine position using a breast board while their left arm was placed above the head. To perform $\mathrm{DIBH}$, a novel system was used as a house respiratory gating system [22]. This system uses the CD22-100AM122 distance meter laser sensor. The laser was adjusted between the xiphoid process and umbilicus at a distance of $10 \mathrm{~cm}$ to the body surface. This distance is changed by respiration, and then, these changes are detected in the output of the sensor. As a result, the respiratory signal is displayed on the monitor. Firstly, the patient was requested to breathe in and out and then breathe deeply. When a stable deep inspiration level was acquired, the upper and lower thresholds were placed on the respiratory signal for the determination of the gating window. Moreover, the gating window was adjusted to $5 \mathrm{~mm}$. Finally, the patient was asked to breathe deeply and put the respiratory signal in the gating window and keep it for approximately 20 seconds; therefore, the CT image was acquired at this time.

\section{Treatment planning}

The clinical target volume (CTV) and OARs, including the heart and left lung, were contoured by an oncologist. The prescribed dose was 50 Gy in 2 Gy daily fractions to the CTV. It was delivered by the three-dimensional conformal technique with two opposing 6 MV tangential fields and additional subfields. In the treatment planning, at least 95\% of the CTV should be covered by the 95\% isodose lines. In addition, hotspots up to $107 \%$ of the prescribed dose were acceptable. After planning, central lung distance (CLD) and lung length (LL) were calculated in the medial field. The CLD refers to the distance between the edge of the lung contour and tangential field border in the central axis on a digitally reconstructed radiograph; however, LL refers to the vertical distance of the lung, which is vertically placed in the radiation port [23].

\section{Image analysis}

In the current study, 3D Slicer software (version 4.10.1) was used for the calculation of the parameters, including the irradiated heart and left lung volumes, as well as the displacement values, for them during $\mathrm{DIBH}$.

\section{Irradiated volumes of heart and left lung}

In this study, the absolute and relative irradiated volumes for the heart and left lung were calculated for FB and DIBH separately. For instance, the method of the calculation of the irradiated left lung volume in DIBH was completely described as it follows.

For this purpose, the CT image, RT Structure, and RT Plan were loaded into the software platform. Then, the Segmentation module was used for the creation of the left lung model. After that, the lung model and radiation fields were imported into the segmentation created by the segmentation module.

The intersection between the left lung model and each of the radiation fields were obtained by the Segment Editor module. The irradiated volumes in the medial and lateral fields were added to- 
gether by which the total left lung volume was acquired in the radiation fields. Finally, the Segment Statistics module was used for the calculation of the numerical value of this irradiated volume. In addition, to estimate the relative irradiated volume, the absolute irradiated volume was divided into the total volume. As shown in Fig. 1, the irradiated volumes of the heart and left lung were calculated in the FB and DIBH states.

\section{Calculation of displacements for heart and left lung during DIBH}

In the present study, the numerical values of the displacement were calculated for the heart and left lung between two FB and DIBH states. For instance, the methods of calculating the magnitude of the left lung displacement and displacement vectors in different directions (i.e., anterior-posterior $[A P]$, right-left $[R L]$, and superior-inferior [SI]) were completely described as it follows.

\section{1) Magnitude of displacement}

Firstly, the CT images and RT Structures were loaded into the software platform. After that, the Segmentation module was used for the creation of the left lung models for FB and DIBH separately. For the calculation of the displacement field, the left lung models had to be registered between the FB and DIBH states using the Segment Registration module. Following that, all displacement magnitudes were obtained by the Transforms module. Finally, the Segment Statistics module was used for the calculation of the average value of displacement magnitudes.

\section{2) Displacement vectors in $S I, A P$, and RL directions}

For obtaining the displacement vectors in the AP, RL, and SI directions, the left lung models were registered as before. After that, the

(A)

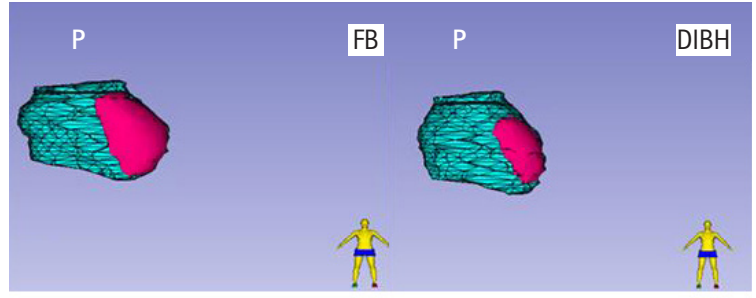

B

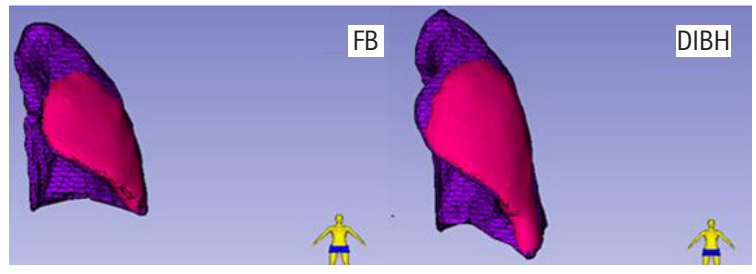

Fig. 1. Irradiated volumes in tangential fields: (A) heart and (B) left lung. $\mathrm{FB}$, free breathing; $\mathrm{DIBH}$, deep inspiration breath hold. deformable left lung was imported into the segmentation created by the Segmentations module. The out regions of the left lung should be blanked to have the displacement vectors only in the deformable left lung. For this purpose, the Segment Editor module was used in this study. The result of this step was displacement vectors only for the deformable left lung renamed to the masked displacement field. The masked displacement field was saved and then extracted its components in the $A P, R L$, and $S I$ directions by a self-written program in Python programming language followed by the calculation of their mean values in any directions. Furthermore, the differences between the parameters in $\mathrm{DIBH}$ and FB plans were calculated for each patient. These differences $(\Delta X)$ were calculated at $X_{D B B H}-X_{F B}$. After that, $\Delta X$ was normalized to the $X_{F B}$ that resulted in $\triangle^{\prime} X$ and expressed as percentage.

\section{Statistical analysis}

A comparison between the parameters for FB and DIBH plans was performed using a paired sample t-test in SPSS version 22.0 (SPSS IBM, Armonk, New York, USA). These parameters included the $D_{\text {mean }}, V_{20}, V_{25}, V_{\text {abs }}, V_{\text {rel }}, C L D$, and LL. In addition, Pearson correlation coefficient was used to determine the correlation between the left lung $\Delta^{\prime} \mathrm{D}_{\text {mean }}$ with the $\Delta^{\prime} \mathrm{V}_{\text {absı }} \Delta^{\prime} \mathrm{V}_{\text {relı }} \Delta^{\prime} \mathrm{CLD}$, and $\Delta^{\prime} \mathrm{LL}$. $\mathrm{p}$-value less than 0.05 was considered statistically significant. Finally, the average of mean displacement magnitudes, as well as vectors in $\mathrm{SI}, \mathrm{AP}$, and $\mathrm{RL}$ directions, were calculated in this study.

\section{Results}

As shown in Table 1, DIBH was associated with a significant reduction in the heart dosimetric parameters ( $p<0.001$ ); however, there was no statistically significant difference in the left lung dosimetric parameters ( $p>0.05$ ). The average of the heart $D_{\text {mean }}$ reduced from 5.97 to 3.83 Gy with DIBH. Similarly, the mean of $V_{25}$ decreased from $7.60 \%$ to $3.29 \%$. The average of left lung $D_{\text {mean }}$ was changed from $8.67 \mathrm{~Gy}(\mathrm{FB})$ to $8.95 \mathrm{~Gy}$ (DIBH). In addition, the mean of $\mathrm{V}_{20}$ was obtained $14.84 \%$ with $\mathrm{FB}$ and $15.44 \%$ by DIBH. The average of CLD significantly increased from 2.18 to $2.47 \mathrm{~cm}$ with DIBH $(p<0.001)$. Moreover, LL significantly increased from $14.12 \mathrm{~cm}$ for FB to $18.05 \mathrm{~cm}$ for DIBH ( $<<0.001)$.

\section{Irradiated volumes of heart and left lung}

The results of Table 1 show that the mean of heart $\mathrm{V}_{\mathrm{abs}}$ significantly decreased from 42.12 to $15.82 \mathrm{~mL}$ using DIBH ( $<0.001)$. Similarly, heart $\mathrm{V}_{\text {rel }}$ decreased from $8.16 \%$ to $3.17 \%$ ( $\left.p<0.001\right)$. For the left lung, $V_{\text {abs }}$ significantly increased from $124.32 \mathrm{~mL}$ with FB to $223.27 \mathrm{~mL}$ by DIBH ( $p<0.001)$. Furthermore, $\mathrm{V}_{\text {rel }}$ was changed from $13.33 \%$ with FB to $13.99 \%$ by DIBH ( $>>0.05$ ). As 
Table 1. Comparison of dosimetric parameters, CLD, and LL between FB and DIBH plans in patients with left-sided breast cancer

\begin{tabular}{lcccr}
\hline Parameter & FB & DIBH & Paired difference relative changes (\%) & $p$-value \\
\hline Heart & & & & \\
$D_{\text {mean }}(\mathrm{Gy})$ & $5.97 \pm 2.0$ & $3.83 \pm 1.40$ & $-2.14,-35.85$ & $<0.001$ \\
$\mathrm{~V}_{25}(\%)$ & $7.60 \pm 4.10$ & $3.29 \pm 2.64$ & $-4.31,-56.71$ & $<0.001$ \\
$\mathrm{~V}_{\text {abs }}(\mathrm{mL})$ & $42.12 \pm 29.54$ & $15.82 \pm 15.46$ & $-26.30,-62.44$ & $<.001$ \\
$\mathrm{~V}_{\text {rel }}(\%)$ & $8.16 \pm 4.62$ & $3.17 \pm 2.69$ & $-4.99,-61.15$ & $<0.001$ \\
Left lung & & & & 0.389 \\
$\mathrm{D}_{\text {mean }}(\mathrm{Gy})$ & $8.67 \pm 2.32$ & $8.95 \pm 1.79$ & $0.28,3.23$ & 0.387 \\
$\mathrm{~V}_{20}(\%)$ & $14.84 \pm 4.97$ & $15.44 \pm 3.85$ & $0.60,4.04$ & $<0.001$ \\
$\mathrm{~V}_{\text {abs }}(\mathrm{mL})$ & $124.32 \pm 55.76$ & $223.27 \pm 79.41$ & $98.95,79.59$ & 0.350 \\
$\mathrm{~V}_{\text {rel }}(\%)$ & $13.33 \pm 5.31$ & $13.99 \pm 4.10$ & $0.66,4.95$ & 0.001 \\
CLD $(\mathrm{cm})$ & $2.18 \pm 0.62$ & $2.47 \pm 0.64$ & $0.29,13.30$ & $<0.001$ \\
LL $(\mathrm{cm})$ & $14.12 \pm 1.83$ & $18.05 \pm 1.73$ & $3.93,27.83$ & \\
\hline
\end{tabular}

Values are presented as mean \pm standard deviation.

$C L D$, central lung distance; $L L$, lung length; $F B$, free breathing; $D I B H$, deep inspiration breath hold; $D_{\text {mean }}$ mean dose; $V X$, relative volume receiving $\geq X$ $G y ; V_{\text {abs }}$ absolute irradiated volume; $V_{\text {relı }}$ relative irradiated volume.

Significant differences with $\mathrm{p}<0.05$.

Table 2. Pearson correlation coefficient between $\Delta^{\prime}$ Dmean and other parameters for left lung

\begin{tabular}{ccccc}
\hline & $\Delta^{\prime} \mathrm{V}_{\text {abs }}(\%)$ & $\Delta^{\prime} \mathrm{V}_{\text {rel }}(\%)$ & $\Delta^{\prime} \mathrm{CLD}(\%)$ & $\Delta^{\prime} \mathrm{LL}(\%)$ \\
\hline$\Delta^{\prime} \mathrm{D}_{\text {mean }}(\%)$ & $r=0.815, \mathrm{p}<0.001$ & $r=0.932, p<0.001$ & $r=0.693, p=0.002$ & $r=0.143, p=0.667$
\end{tabular}

$\Delta^{\prime} X(\%)$, percentage of changes in $X$ parameter; $D_{\text {mean }}$ mean dose; $V_{\text {abs }}$ absolute irradiated volume; $V_{\text {relı }}$ relative irradiated volume; $C L D$, central lung distance; LL, lung length.

Significant correlations with $p<0.05$.

shown in Table 2, there was a significant correlation between $\Delta^{\prime} \mathrm{D}$ with both of the $\Delta^{\prime} \mathrm{V}_{\text {abs }}$ and $\Delta^{\prime} \mathrm{V}_{\text {rel }}$. Moreover, the correlation between the $\Delta^{\prime} \mathrm{D}$ and $\Delta^{\prime} \mathrm{CLD}$ was significant $(\mathrm{p}=0.002)$; however, the correlation between $\Delta^{\prime} \mathrm{D}$ and $\Delta^{\prime}$ 'LL was not significant $(\mathrm{p}=$ 0.667).

\section{Displacements for heart and left lung during DIBH}

As shown in Table 3, the average of mean displacement magnitude for the heart was $7.32 \mathrm{~mm}$. In addition, the maximum value for the mean of heart displacement vectors was in the inferior direction. The average of the mean displacement magnitude for the left lung was $20.91 \mathrm{~mm}$. Moreover, the maximum values for the mean of left lung displacement were in the SI directions.

\section{Discussion and Conclusion}

The results of the present study demonstrated the ability of DIBH in the reduction of the heart dose in patients with left-sided breast cancer as reported in previous studies [7-12]. However, similar to some reports, the collected data of the present study did not show the protective effect of this technique on the left lung $[8,15-20]$. In the present study, the average of the heart $D_{\text {mean }}$ and
Table 3. Average of mean displacement magnitude and mean displacement vectors in different directions

\begin{tabular}{lcr}
\hline Parameter & Heart & \multicolumn{1}{c}{ Left lung } \\
\hline Mean of displacement magnitude & $7.32 \pm 2.45$ & $20.91 \pm 5.03$ \\
Displacement direction & & \\
Mean $_{\text {Right }}(\mathrm{mm})$ & $3.33 \pm 1.83$ & $7.55 \pm 3.02$ \\
$\operatorname{Mean}_{\text {Left }}(\mathrm{mm})$ & $3.15 \pm 1.47$ & $4.70 \pm 1.25$ \\
$\operatorname{Mean}_{\text {Anterior }}(\mathrm{mm})$ & $1.93 \pm 1.06$ & $10.00 \pm 2.87$ \\
$\operatorname{Mean}_{\text {Posterior }}(\mathrm{mm})$ & $2.43 \pm 1.17$ & $5.03 \pm 2.30$ \\
$\operatorname{Mean}_{\text {Superior }}(\mathrm{mm})$ & $2.12 \pm 1.75$ & $14.61 \pm 5.22$ \\
$\operatorname{Mean}_{\text {Inferior }}(\mathrm{mm})$ & $4.89 \pm 1.73$ & $13.66 \pm 5.36$ \\
\hline
\end{tabular}

Values are presented as mean \pm standard deviation.

Mean $\mathrm{X}$, average of mean displacement in $\mathrm{X}$ direction.

$\mathrm{V}_{25}$ significantly decreased using DIBH. Also, the absolute and relative irradiated volumes of the heart significantly decreased by this technique. In DIBH, the left lung expansions in different directions pushed the heart away from the radiation fields and irradiated areas that is the most important reason for the reduction of irradiated heart volume and dose.

Jensen et al. [24] reported that the average of the heart $D_{\text {mean }}$ de- 
creased from 3 Gy with FB to 2 Gy by DIBH. In addition, they observed that the average of the heart $\mathrm{V}_{25}$ decreased from 3.3\% (FB) to $1.0 \%$ (DIBH). Sung et al. [25] demonstrated that the average of the heart $\mathrm{D}_{\text {mean }}$ decreased from $5.9 \mathrm{~Gy}$ with $\mathrm{FB}$ to $3 \mathrm{~Gy}$ in DIBH.

Hayden et al. [8] reported that the irradiated heart volumes were $36.9 \mathrm{~mL}$ (FB) and $2.1 \mathrm{~mL}$ (DIBH), which were smaller than the results of the present study. In addition, in a study carried out by Sung et al. [25], the irradiated heart volumes were reported as 41.7 and $12 \mathrm{~mL}$ for FB and DIBH, respectively, which were almost consistent with the findings of the present study. The differences between contouring and patient anatomy lead to higher dosimetric parameters of the heart, compared to those in other studies. The smaller lung volume in Asian women in comparison to that in non-Asians might lead to a lower distance between the left ventricle and chest wall, which explains the higher irradiated heart volume in this study $[7,26]$.

The maximum value for the mean of heart displacement vectors was in the inferior direction due to lung inflation and diaphragmatic movement that results in the greatest heart displacements in this direction.

Although the results of previous studies have shown that the mean heart dose reduces with DIBH [7-12], there are still some discrepancies in the left lung dosimetric parameters using this technique. In some studies, it was demonstrated that lung $D_{\text {mean }}$ significantly reduced with DIBH $[2,7,11,14]$. However, in some studies, the lung dose was not significantly changed or even increased $[8,15-20]$. In the present study, it was observed that using DIBH led to an increase in the lung dose for some patients.

The obtained results of the present study showed that the absolute and relative irradiated volumes of the left lung increased using $\mathrm{DIBH}$; however, the increase of relative irradiated volume was not significant. Also, Sung et al. [25] demonstrated that the average of irradiated lung volume increased from $155.1 \mathrm{~mL}$ (FB) to $253.5 \mathrm{~mL}$ (DIBH). Although, the results of some studies have shown that in the DIBH technique, the relative irradiated volume decreases despite increasing the absolute irradiated lung volume [2,12], Nevertheless, the results of the present study revealed that the relative irradiated volume increased in some patients. This volume was higher in DIBH than that in FB in almost all patients of the present study whose lung dosimetric parameters increased with $\mathrm{DIBH}$. Therefore, it is expected to increase the dosimetric parameters of the left lung, if the relative irradiated volume increases.

Overall, discrepancies in the left lung dosimetric parameters among the studies are related to various factors, such as differences in the patient anatomy, contouring, treatment planning, and treatment methods.

The CLD is a parameter that can be used for the prediction of the irradiated lung volume in the tangential fields. The CLDs of 1.5, 2.5 , and $3.5 \mathrm{~cm}$ can approximately predict 6\%, 16\%, and 26\% of the ipsilateral lung, respectively, that would be included in the tangential fields [27]. The results of the patients in the present study were almost consistent with these values. Furthermore, the average of mean displacement of left lung magnitude in the present study was $20.91 \mathrm{~mm}$. In addition, the maximum values of the mean displacement were acquired in SI directions and following that anterior direction.

In a study conducted by Oechsner et al. [28], the mean of displacement magnitude for the left lung was reported as $20.80 \mathrm{~mm}$. Moreover, they calculated the mean of displacement in three directions, including left $(1.5 \mathrm{~mm})$, anterior $(16.0 \mathrm{~mm})$, and inferior $(12.2 \mathrm{~mm})$. According to the results of the present study and collected data, the averages of the left lung displacement magnitude in the two studies are completely similar. Nevertheless, in the aforementioned study, the anterior displacement is greater than the other two directions. However, in the present study, the maximum values of the mean displacement vectors were acquired in the superior and inferior directions and after that in the anterior direction. Their patients were trained to do chest breathing, although the patients in the present study were not trained to perform specific breathing that is an important reason for differences between the results of the two studies.

According to the potential factors that result in the increased left lung dosimetric parameters using $\mathrm{DIBH}$, it seems that lung displacement into the irradiated areas is the most important factor. As shown in Fig. 2, the anterior displacement of the lung had the greatest effect on displacement toward the CTV. In addition, while performing $\mathrm{DIBH}$, the parts of the lung outside the radiation field are placed in the field, as shown in Fig. 3. When the left lung moves toward the radiation fields, the absolute irradiated volume, as well as, the relative irradiated volume, increased, which resulted in an increase in the lung dosimetric parameters.

As shown in Table 2, by assessing the correlation of the lung $\Delta^{\prime} \mathrm{D}$ with other factors, such as $\Delta^{\prime} \mathrm{V}_{\mathrm{abs}} \Delta^{\prime} \mathrm{V}_{\text {rel, }} \Delta^{\prime} \mathrm{CLD}$, and $\Delta^{\prime} \mathrm{LL}$, there was a significant correlation between $\Delta^{\prime} \mathrm{D}$ with both $\Delta^{\prime} \mathrm{V}_{\text {abs }}$ and $\Delta^{\prime} \mathrm{V}_{\text {rel- }}$. The lung dosimetric parameters, such as $D_{\text {mean }}$ and $V_{20}$, can increase by rising the irradiated lung volume, especially $V_{\text {rel }}$. Moreover, there was a significant correlation between $\triangle^{\prime} \mathrm{D}$ and $\triangle^{\prime} C L D$. As previously mentioned, CLD is associated with irradiated lung volume. The increase in the CLD leads to an increase in lung $\mathrm{V}_{\mathrm{abs}}$ and consequently $V_{\text {rel }}$ for some patients. Therefore, lung $D_{\text {mean }}$ increased in these cases. However, the significant correlation was not observed between $\Delta^{\prime} \mathrm{D}$ and $\Delta^{\prime} \mathrm{LL}$. It is argued that among some patients in the present study, the left lung cranio-caudal movement had no significant effect on their dose reduction, and the 

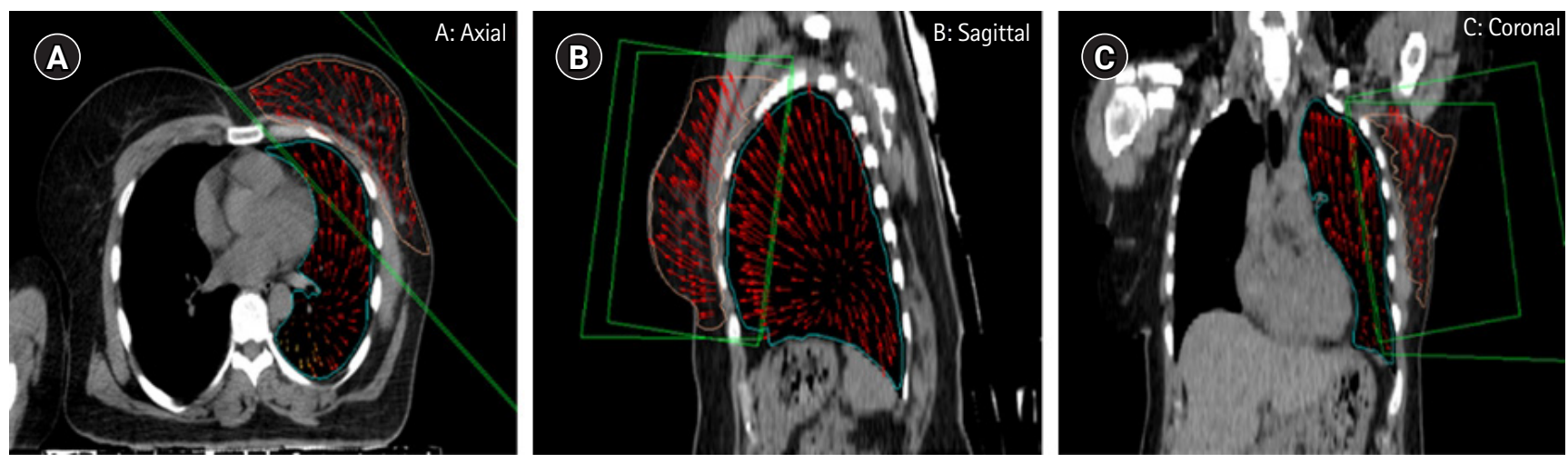

Fig. 2. Representation of displacement vectors for one of the patients with increasing left lung dosimetric parameters during deep inspiration breath hold: (A) axial, (B) sagittal, and (C) coronal.
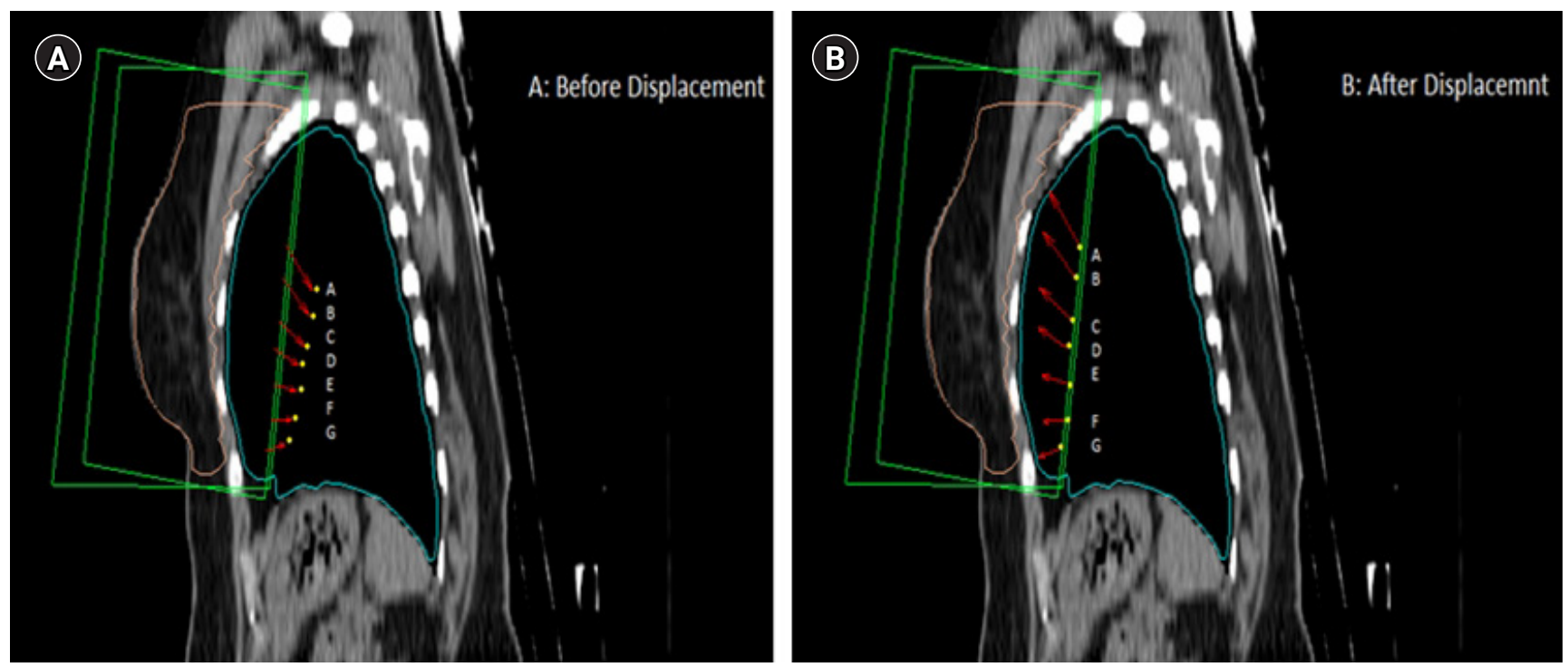

Fig. 3. The A-G point positions of the left the lung relative to the radiation fields: (A) before displacement and (B) after displacement.

anterior displacement was a more important factor in lung dose. Even in some of them, the upward and downward movements were greater than the anterior ones.

In addition, special attention should be given to patient anatomy that can be considered an effective factor in lung dose. Factors such as breast size and CTV, which determine the size of the radiation fields, as well as the chest shape can be considered effective factors in the lung dose. As Chilukuri et al. [29] reported in their abstract that the mean of the lung $\mathrm{V}_{20}$ significantly decreased in patients with a curved chest wall (12\% vs. $19 \%$; $p=0.001)$; however, the mean of lung $V_{20}$ was acquired $21 \%$ versus $22.3 \%$ in patients with a flat chest wall ( $p=0.78$ ). According to the heart and lung dosimetric parameters, they concluded that patients with a curved chest wall obtained a significant benefit from DIBH.

In general, with respect to the dosimetric parameters of the heart in the present study, all of the patients in this study could benefit from the DIBH technique, especially the subjects with large irradiated heart volume in the FB plan. Nevertheless, the results of the current study revealed that the DIBH technique did not necessarily decrease the lung dose. Based on the various factors, such as patient anatomy, including chest shape, breast size, direction of the lung displacements, as well as contouring and treatment planning, the lung dose can be varied in different patients, as shown in Fig. 4.

Considering the heart dosimetric parameters, Joo et al. [7] reported that patients with long maximum heart distance (MHD) and small lung volume have highly benefited from this technique. However, it is required to perform further studies for the determination of the anatomical parameters that affect both the heart and lung dose reduction and lead to the selection of patients most 

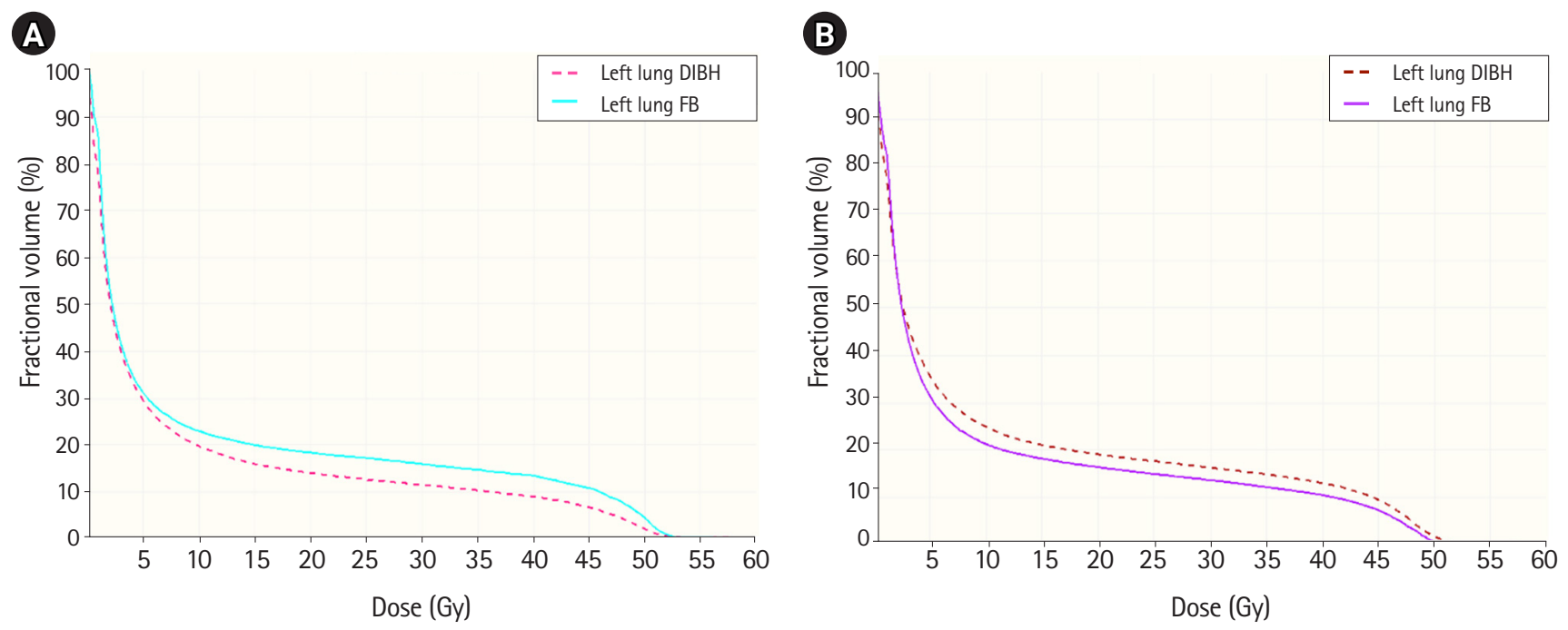

Fig. 4. Left lung dose-volume histograms for 2 patients: (A) dose reduction and (B) increasing the lung dose by deep inspiration breath hold (DIBH). FB, free breathing.

likely to benefit from the DIBH technique.

Another strategy for the improvement of heart sparing in breast cancer treatment is intensity-modulated radiation therapy. In this technique, due to using more radiation fields, the normal volumes that occur in the radiation fields increase, which results in the increase of the normal tissue low-dose exposure. In addition, in this technique, due to the healthy tissues and

CTV proximity, normal tissues occur in most radiation fields. Despite the reduction in the high-dose area, the low- and medium-dose regions in the heart and lung increase $[2,30]$ that can raise the dosimetric parameters of OARs for some patients, as reported in some studies [31-34]. However, the high-dose regions in the heart could decrease without these additional increases in the low- and medium-dose areas using DIBH [2]. Nonetheless, the lung dose can be different in various patients with this technique.

As a result, in comparison to $\mathrm{FB}, \mathrm{DIBH}$ is a more effective technique for the reduction of the heart dose, which is sometimes associated with increasing the left lung dosimetric parameters.

\section{Conflicts of interest}

No potential conflict of interest relevant to this article was reported.

\section{Acknowledgements}

The authors would like to thank the Research Vice-Chancellor of Mashhad University of Medical Sciences, Mashhad, Iran, for financial support from 970830 protocol code.

\section{References}

1. Early Breast Cancer Trialists' Collaborative Group (EBCTCG). Effect of radiotherapy after breast-conserving surgery on 10-year recurrence and 15-year breast cancer death: meta-analysis of individual patient data for 10,801 women in 17 randomised trials. Lancet 2011;378:1707-16.

2. Vikstrom J, Hjelstuen MH, Mjaaland I, Dybvik KI. Cardiac and pulmonary dose reduction for tangentially irradiated breast cancer, utilizing deep inspiration breath-hold with audio-visual guidance, without compromising target coverage. Acta Oncol 2011; 50:42-50.

3. Darby SC, McGale P, Taylor CW, Peto R. Long-term mortality from heart disease and lung cancer after radiotherapy for early breast cancer: prospective cohort study of about 300,000 women in US SEER cancer registries. Lancet Oncol 2005;6:557-65.

4. Darby SC, Ewertz M, McGale P, et al. Risk of ischemic heart disease in women after radiotherapy for breast cancer. $\mathrm{N}$ Engl J Med 2013;368:987-98.

5. Rochet N, Drake Jl, Harrington K, et al. Deep inspiration breathhold technique in left-sided breast cancer radiation therapy: Evaluating cardiac contact distance as a predictor of cardiac exposure for patient selection. Pract Radiat Oncol 2015;5:e127-34.

6. Duma MN, Münch S, Oechsner M, Combs SE. Heart-sparing radiotherapy in patients with breast cancer: What are the techniques used in the clinical routine? A pattern of practice survey in the German-speaking countries. Med Dosim 2017;42:197202.

7. Joo JH, Kim SS, Ahn SD, et al. Cardiac dose reduction during tan- 
gential breast irradiation using deep inspiration breath hold: a dose comparison study based on deformable image registration. Radiat Oncol 2015;10:264.

8. Hayden AJ, Rains M, Tiver K. Deep inspiration breath hold technique reduces heart dose from radiotherapy for left-sided breast cancer. J Med Imaging Radiat Oncol 2012;56:464-72.

9. Saini AS, Hwang CS, Biagioli MC, Das IJ. Evaluation of sparing organs at risk (OARs) in left-breast irradiation in the supine and prone positions and with deep inspiration breath-hold. J Appl Clin Med Phys 2018;19:195-204.

10. Lin A, Sharieff W, Juhasz J, Whelan T, Kim DH. The benefit of deep inspiration breath hold: evaluating cardiac radiation exposure in patients after mastectomy and after breast-conserving surgery. Breast Cancer 2017;24:86-91.

11. Schonecker $S$, Walter $F$, Freislederer $P$, et al. Treatment planning and evaluation of gated radiotherapy in left-sided breast cancer patients using the Catalyst(TM)/Sentinel(TM) system for deep inspiration breath-hold (DIBH). Radiat Oncol 2016;11:143.

12. Bruzzaniti V, Abate A, Pinnaro P, et al. Dosimetric and clinical advantages of deep inspiration breath-hold (DIBH) during radiotherapy of breast cancer. J Exp Clin Cancer Res 2013;32:88.

13. Chan TY, Tang Jl, Tan PW, Roberts N. Dosimetric evaluation and systematic review of radiation therapy techniques for early stage node-negative breast cancer treatment. Cancer Manag Res 2018;10:4853-70.

14. Hepp R, Ammerpohl M, Morgenstern $C$, et al. Deep inspiration breath-hold (DIBH) radiotherapy in left-sided breast cancer: dosimetrical comparison and clinical feasibility in 20 patients. Strahlenther Onkol 2015;191:710-6.

15. Bartlett FR, Donovan EM, McNair HA, et al. The UK HeartSpare Study (Stage II): multicentre evaluation of a voluntary breathhold technique in patients receiving breast radiotherapy. Clin Oncol (R Coll Radiol) 2017;29:e51-e56.

16. Frazier RC, Vicini FA, Sharpe MB, et al. Impact of breathing motion on whole breast radiotherapy: a dosimetric analysis using active breathing control. Int J Radiat Oncol Biol Phys 2004; 58:1041-7.

17. Walston S, Quick AM, Kuhn K, Rong Y. Dosimetric considerations in respiratory-gated deep inspiration breath-hold for left breast irradiation. Technol Cancer Res Treat 2017;16:22-32.

18. Mast ME, van Kempen-Harteveld $L$, Heijenbrok MW, et al. Left-sided breast cancer radiotherapy with and without breathhold: does IMRT reduce the cardiac dose even further? Radiother Oncol 2013;108:248-53.

19. Osei $E_{1}$ Darko J, Fleck $A$, et al. Dosimetric evaluation of wholebreast radiation therapy: clinical experience. Med Dosim 2015; 40:355-65.
20. Rice L, Goldsmith C, Green MM, Cleator S, Price PM. An effective deep-inspiration breath-hold radiotherapy technique for leftbreast cancer: impact of post-mastectomy treatment, nodal coverage, and dose schedule on organs at risk. Breast Cancer (Dove Med Press) 2017:9:437-46.

21. Fedorov A, Beichel R, Kalpathy-Cramer J, et al. 3D Slicer as an image computing platform for the Quantitative Imaging Network. Magn Reson Imaging 2012;30:1323-41.

22. Farzaneh MJK, Nasseri S, Momennezhad M, Salek R. Design and construction of a laser-based respiratory gating system for implementation of deep inspiration breathe hold technique in radiotherapy clinics. J Med Signals Sens 2018;8:253-62.

23. Kong FM, Klein EE, Bradley JD, et al. The impact of central lung distance, maximal heart distance, and radiation technique on the volumetric dose of the lung and heart for intact breast radiation. Int J Radiat Oncol Biol Phys 2002;54:963-71.

24. Jensen CA, Abramova T, Frengen J, Lund JA. Monitoring deep inspiration breath hold for left-sided localized breast cancer radiotherapy with an in-house developed laser distance meter system. J Appl Clin Med Phys 2017;18:117-23.

25. Sung K, Lee KC, Lee SH, Ahn SH, Lee SH, Choi J. Cardiac dose reduction with breathing adapted radiotherapy using self respiration monitoring system for left-sided breast cancer. Radiat Oncol J 2014;32:84-94.

26. Yang TS, Peat J, Keena V, Donnelly P, Unger W, Woolcock A. A review of the racial differences in the lung function of normal Caucasian, Chinese and Indian subjects. Eur Respir J 1991;4:87280.

27. Bornstein BA, Cheng CW, Rhodes LM, et al. Can simulation measurements be used to predict the irradiated lung volume in the tangential fields in patients treated for breast cancer? Int J Radiat Oncol Biol Phys 1990;18:181-7.

28. Oechsner M, Dusberg M, Borm KJ, Combs SE, Wilkens JJ, Duma MN. Deep inspiration breath-hold for left-sided breast irradiation: analysis of dose-mass histograms and the impact of lung expansion. Radiat Oncol 2019;14:109.

29. Chilukuri S, Adulkar D, Subramaniam S, et al. P0-0881: patient selection for DIBH technique for left sided breast cancers: impact of chest wall shape. Radiat Oncol 2016;119:S422.

30. Swanson T, Grills IS, Ye H, et al. Six-year experience routinely using moderate deep inspiration breath-hold for the reduction of cardiac dose in left-sided breast irradiation for patients with early-stage or locally advanced breast cancer. Am J Clin Oncol 2013; 36:24-30.

31. Haciislamoglu $E_{1}$ Colak $F_{1}$ Canyilmaz $E_{1}$ et al. The choice of multibeam IMRT for whole breast radiotherapy in early-stage right breast cancer. Springerplus 2016;5:688. 
32. Haciislamoglu $E_{1}$ Colak F, Canyilmaz $E_{1}$ et al. Dosimetric comparison of left-sided whole-breast irradiation with 3DCRT, forward-planned IMRT, inverse-planned IMRT, helical tomotherapy, and volumetric arc therapy. Phys Med 2015;31:360-7.

33. Jin GH, Chen LX, Deng XW, Liu XW, Huang Y, Huang XB. A comparative dosimetric study for treating left-sided breast cancer for small breast size using five different radiotherapy techniques: conventional tangential field, filed-in-filed, tangential-IMRT, multi-beam IMRT and VMAT. Radiat Oncol 2013;8:89.

34. Liu H, Chen $X$, He Z, Li J. Evaluation of 3D-CRT, IMRT and VMAT radiotherapy plans for left breast cancer based on clinical dosimetric study. Comput Med Imaging Graph 2016;54:1-5. 